\title{
CLINICAL RESEARCH ARTICLE Osteopontin in human milk and infant formula affects infant plasma osteopontin concentrations
}

\author{
Rulan Jiang $^{1}$ and Bo Lönnerdal ${ }^{1}$
}

\begin{abstract}
BACKGROUND: Osteopontin (OPN), a multifunctional protein, is present abundantly in human milk, but not in bovine milk and infant formulas. A recent randomized clinical trial showed that supplementing infant formula with bovine milk OPN (bOPN) resulted in better immune outcomes.

METHODS: Human milk OPN (hOPN) concentrations were analyzed by ELISA. Plasma samples were obtained from infants receiving one of four treatments: breast milk (BF), unsupplemented formula (F0), formula supplemented with 65 mg/L bOPN (F65), or with $130 \mathrm{mg} / \mathrm{L}$ bOPN (F130). Plasma samples were analyzed for hOPN and bOPN by ELISA.

RESULTS: The hOPN concentration was high in early lactation (D1 to D8), decreased gradually after D9, and deceased significantly after 1 month. At 4 and 6 months, higher levels of hOPN were found in plasma samples from the BF, F65, and F130 groups than in samples from the F0 group; the plasma bOPN concentration in the F130 group was greater than that in the F65 group.

CONCLUSION: Dynamic changes in the concentration of milk OPN may reflect infant needs for different amounts of milk OPN for various functions at different developmental stages. Supplemental bOPN in infant formula may exert its beneficial effects by increasing endogenous OPN in plasma.
\end{abstract}

Pediatric Research (2019) 85:502-505; https://doi.org/10.1038/s41390-018-0271-x

\section{INTRODUCTION}

Osteopontin (OPN) is a highly post-translationally modified glycoprotein with multiple functions including immunomodulatory activities, tissue remodeling, bone formation, as well as cell attachment, migration, proliferation, and differentiation. ${ }^{1,2}$ OPN contains RGD and non-RGD integrin binding domains, ${ }^{3-6}$ as well as a CD44-binding motif, 7,8 and OPN exerts its multiple functions by binding to its receptors on cell membranes to initiate signaling cascades. ${ }^{9}$ OPN is synthesized by a variety of tissues and appears in almost all body fluids, including breast milk ${ }^{10}$ where it is present at a high concentration, $\sim 138 \mathrm{mg} / \mathrm{L}$ in milk from early lactation [mean: 20 days postpartum milk samples collected in Denmark ${ }^{11}$ ] and $157 \mathrm{mg} / \mathrm{L}$ later in lactation [median of 3.0-19.3 weeks postpartum milk samples collected in China, Denmark, Japan, and Korea ${ }^{12}$, making it one of the five most abundant breast milk proteins. ${ }^{13}$ By incubating bOPN with human neonatal gastric juice an in vitro study showed that bOPN is resistant to gastric digestion, ${ }^{14}$ and a study in 10-week-old mice showed that ingested bOPN increased plasma OPN levels. ${ }^{15}$ Milk OPN may thus significantly contribute to development in early infancy. Compared to human milk, bovine milk and infant formula contain significantly less OPN: $\sim 18$ and $\sim 9 \mathrm{mg} / \mathrm{L}$ in bovine milk and infant formula, respectively. ${ }^{11}$ Since in vitro and in vivo studies previously showed that bOPN (Lacprodan OPN-10, Arla Foods Ingredients, Viby, Denmark) caused no adverse effects in bacteria (Salmonella typhimurium strains, and an Escherichia coli strain), human lymphocytes, and animals (7-13 weeks old mice, adult rats, and pregnant rats), ${ }^{16}$ we recently conducted a clinical trial to examine the effects of supplementing infant formula with bOPN at levels similar to hOPN found in breast milk. ${ }^{17,18}$ The bOPN formulas were well tolerated, and we found that infants fed formula with supplemental bOPN (65 or $130 \mathrm{mg} / \mathrm{L}$ ) exhibited lower serum levels of inflammatory cytokines ${ }^{17}$ and an immune cell profile more similar to that of breast-fed infants than to that of infants fed regular formula. ${ }^{18}$ That these findings have functional consequences was supported by a significantly lower prevalence of fever in infants fed OPN-supplemented formula. ${ }^{17}$ However, how the supplemented bOPN exerted its functions remains to be determined.

The present study was undertaken to determine how concentrations of hOPN in breast milk change over a 1-year lactation period and to determine the effect of different feeding regimens on levels of OPN in infant plasma. The OPN content of breast milk was determined at intervals from early lactation (D1) to 12 months of lactation. Infant plasma samples were obtained at 1, 4, and 6 months of age in our previous clinical trial; ${ }^{17}$ infants were breastfed (BF group) or received one of the following diets: unsupplemented formula (F0 group), formula supplemented with $65 \mathrm{mg} / \mathrm{L}$ bOPN (F65 group), or formula supplemented with $130 \mathrm{mg} / \mathrm{L}$ bOPN (F130 group). Plasma samples were analyzed for content of hOPN and bOPN, and the effect of supplemental bOPN on plasma hOPN levels was determined.

\section{METHODS}

Breast milk collection

This study was approved by the Institutional Review Board at the University of California, Davis. Breast milk samples were collected from 12 healthy mothers who exclusively breast-fed their infants in Davis, CA at postpartum days 1-14, and months 1, 2, 4, 6, and 12 of lactation. Only mothers who delivered full-term infants (37-42 weeks) were recruited for the study. One breast was

${ }^{1}$ Department of Nutrition, University of California, Davis, CA 95616, USA

Correspondence: Bo Lönnerdal (bllonnerdal@ucdavis.edu)

Received: 10 September 2018 Revised: 28 November 2018 Accepted: 12 December 2018

Published online: 12 January 2019 
Table 1. hOPN concentrations in milk from different lactation stages

Stage Concentration ( $\mathrm{mg} / \mathrm{L}$,

\begin{tabular}{lc} 
& means \pm SD) \\
\hline Colostrum (D1-D7) & $178.0 \pm 17.9$ \\
Transitional milk (D8-D14) & $134.8 \pm 18.5$ \\
1 month & $65.8 \pm 13.7$ \\
4 months & $48.8 \pm 12.0$ \\
6 months & $55.9 \pm 13.8$ \\
12 months & $48.3 \pm 10.2$ \\
\hline
\end{tabular}

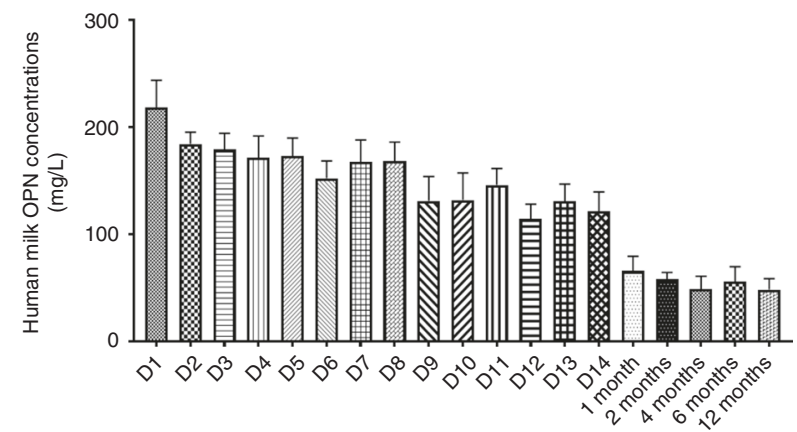

Fig. 1 hOPN concentrations in milk samples. Human OPN concentrations in breast milk samples were measured using an ELISA kit (R\&D Systems) following the manufacturer's instructions. Results are shown as means $\pm S D, n=10-12$ at each time point

emptied completely at the second feeding of the day, and milk samples were stored in $\mathrm{a}-20^{\circ} \mathrm{C}$ freezer until the analysis.

Infant plasma collection

Plasma was isolated from blood samples obtained from 1-, 4-, and 6 -month-old infants in a clinical trial in China ${ }^{17}$ in which infants were recruited at $<1$ month of age and were either exclusively breast-fed (BF group) or fed one of the following formulas: unsupplemented formula (F0 group), formula supplemented with $65 \mathrm{mg} / \mathrm{L}$ bOPN (F65 group), or formula supplemented with $130 \mathrm{mg} / \mathrm{L}$ bOPN (F130 group) up to 6 months of age. bOPN was obtained from Arla Foods Ingredients (Viby, Denmark). The three infant formulas were manufactured by Laiterie de Montaigu (Montaigu, France) using Biostime Premium (marketed by Biostime Inc, Guangzhou, China) as the F0 formula and as the base for the OPN-supplemented formulas. As previously described ${ }^{17}$ blood samples were obtained by venipuncture, and plasma was separated by centrifugation and stored in a $-80^{\circ} \mathrm{C}$ freezer until transfer to UC Davis on dry ice for analysis.

\section{OPN assays}

Human OPN (hOPN) concentrations in breast milk and in all infant plasma samples were measured by an ELISA kit (Human Osteopontin DuoSet ELISA, R\&D Systems, Minneapolis, MN) following the manufacturer's instructions. bOPN was also measured in plasma samples by an ELISA kit (Bovine Osteopontin ELISA, LifeSpan BioSciences, Seattle, WA) following the manufacturer's instructions. There was no cross-reactivity between the two kits; i.e., the hOPN ELISA did not recognize bOPN nor did the bOPN ELISA recognize hOPN.

Data analysis

Data are shown as means \pm standard deviations. Comparisons among treatments and the control were conducted using oneway ANOVA (Prism GraphPad, Berkeley, CA). $p<0.05$ was considered statistically significant.

\section{RESULTS}

Dynamic changes in OPN in breast milk during 12 months of lactation

Colostrum, transitional milk, and mature milk are generally defined as milk produced within 0-7 days postpartum, 8-14 days postpartum, and after 14 days postpartum, respectively. The highest concentration of OPN appeared in D1 milk (Table 1 and Fig. 1). The milk OPN concentration remained relatively stable from D2 to D8, decreased at D9, remained stable from D9 to D14, and then significantly decreased after 1 month and remained constant until the 12th month of lactation. Milk OPN thus is highest in colostrum $(178.0 \pm 17.9 \mathrm{mg} / \mathrm{L})$ and gradually decreases throughout one year of lactation $(48.3 \pm 10.2 \mathrm{mg} / \mathrm{L}$ at 12 months of lactation).

OPN concentrations in infant plasma

As shown in Fig. 2, plasma hOPN concentrations dynamically changed from 1 month to 6 months of age and were highest at 1 month of age for all treatment groups, which may indicate need for different levels of endogenous OPN for different functions during different development stages. At 1 month of age, no significant differences were found among the four infant groups. There was a trend toward lower OPN levels in the infants fed unsupplemented formula, which may be explained by the fact that some infants in the F65 and F130 groups had started receiving the study formulas a few days prior to 1 month of age. In samples from 4 months old infants, plasma hOPN concentrations were greater in the BF, F65, and F130 groups than in the F0 infant group, and no significant differences were seen among BF, F65, and F130 infants. At 6 months of age, BF infants had the highest plasma hOPN concentration, and F0 infants had the lowest hOPN concentration. No differences in hOPN concentrations in plasma were found between the F65 and F130 groups from 1 to 6 months of age.

To examine whether dietary OPN can be transferred to plasma, bOPN concentrations were measured using an ELISA kit. As shown in Fig. 3, low concentrations of bOPN were detected in plasma from infants fed bOPN-supplemented formula (F65 and F130 groups), but not in breast-fed infants (BF group) or infants fed unsupplemented formula (F0 group). Based on a comparison between the concentration of bOPN (Fig. 3) and hOPN in plasma (Fig. 2), the increased hOPN in BF, F65, and F130 plasma samples was not directly due to dietary OPN, an observation that suggests that dietary OPN may promote synthesis or secretion of endogenous OPN. At any given time point, the amount of bOPN in circulation was $<5 \%$ of the hOPN found in plasma of the breastfed infants in this study.

\section{DISCUSSION}

In our longitudinal study, the finding of dynamic changes in the concentration of hOPN in breast milk during 12 months of lactation may indicate infant need for different amounts of OPN for various functions at different developmental stages. A similar trend in hOPN levels was recently reported in a cross-sectional study, in which concentrations of milk OPN were evaluated in milk samples collected from 3 to 20 weeks postpartum in 4 countries (China, Denmark, Japan, and Korea). ${ }^{12}$ It is known that OPN plays important roles in immune modulatory functions and cellmediated immunity, ${ }^{1,19}$ and thus provides protection against pathogens from the surrounding environment. The highest concentrations of milk OPN appear in colostrum and milk from early lactation $(\sim 160-220 \mathrm{mg} / \mathrm{L})$, which may help neonates to adapt to the transition from the intrauterine environment to a complex external environment where they need protection against pathogens. The comparatively lower levels of OPN in transitional $(\sim 130 \mathrm{mg} / \mathrm{L})$ and mature milk $(\sim 50 \mathrm{mg} / \mathrm{L})$ may reflect different roles during later developmental stages. Lactoferrin, 

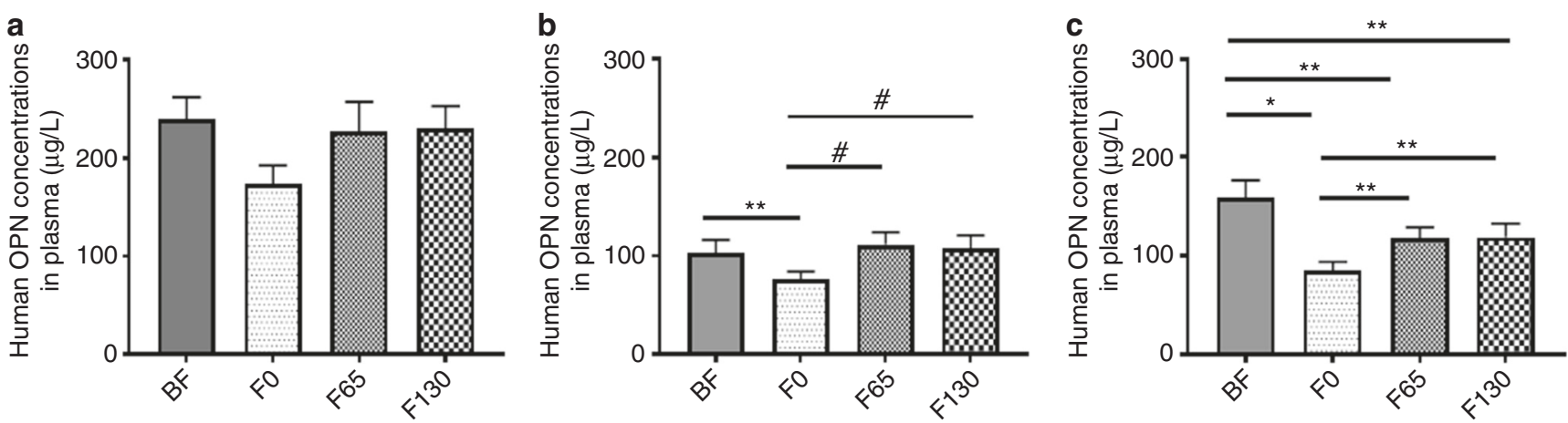

Fig. 2 hOPN concentrations in plasma samples. Human OPN concentrations in all infant plasma samples were measured using a hOPN ELISA kit (R \& D Systems) following the manufacturer's instruction. Plasma samples collected from 1-, 4-, and 6 months old infants (BF, F0, F65, and F130) are shown in $\mathbf{a}, \mathbf{b}$, and $\mathbf{c}$, respectively. Results are shown as means $\pm \mathrm{SD}, n=25$ for each treatment group at each time point, ${ }^{*} p<0.001$, ${ }^{\#} p<0.05,{ }^{* *} p<0.01$

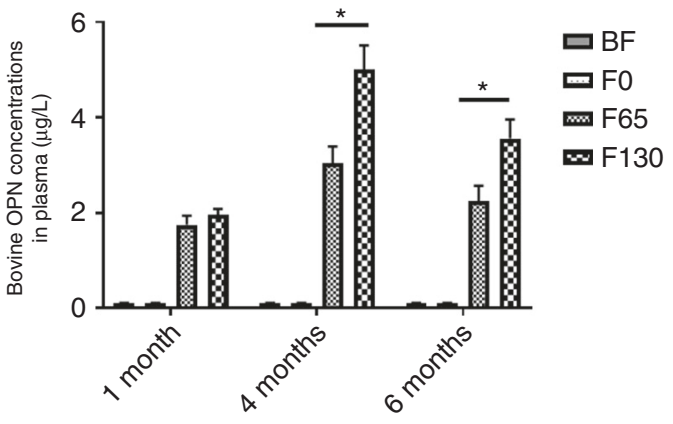

Fig. 3 bOPN concentrations in plasma samples. The bovine OPN concentration in plasma from 1-, 4-, and 6 months old infants (BF, F0, F65, and F130) was measured using a bovine OPN ELISA kit (Lifespan Biosciences) according to the manufacturer's instruction. Results are shown means $\pm S D, n=8$ for each treatment group at each time point. ${ }^{*} p<0.001$

another major milk protein that decreases in concentration during lactation, has also been found to have different functions at different concentrations. ${ }^{20}$ It has been shown in a human enterocyte model (Caco-2 cells) that high concentrations of milk Lf $(1-1000 \mu \mathrm{g} / \mathrm{mL})$, similar to those in milk from early lactation, stimulated intestinal cell proliferation, whereas lower concentrations of milk Lf $(1-1000 \mathrm{ng} / \mathrm{mL})$, such as found in later lactation, enhanced intestinal differentiation. ${ }^{20}$ It is possible that high concentrations of OPN in colostrum and early milk exert biological activities important in early life and that lower intake of OPN in later infancy is adequate to provide other physiological benefits.

In addition to its effect on immune function, milk-derived OPN also affects intestinal development. ${ }^{21}$ In 3-month-old infant monkeys, the intestinal transcriptomes of those that had been breast-fed or received bOPN-supplemented formula (bOPN $125 \mathrm{mg} / \mathrm{L}$ ) were similar, whereas the transcriptome of those receiving unsupplemented formula differed. Our recent study in mice showed that milk OPN promotes brain development by enhancing myelination in early infancy. ${ }^{22}$ In this study, wild-type (WT) mouse pups were nursed from postnatal days 1 to 21 by WT or OPN knock-out (KO) dams that produced milk with high concentrations vs. no OPN, respectively. In addition to showing enhanced myelination, WT mouse pups nursed by WT dams also exhibited better memory and learning ability compared with mouse pups nursed by OPN KO dams.

In the present study, we found that relative to infants fed unsupplemented formula (the F0 group), those infants fed breast milk (the BF group) or bOPN-supplemented formula (the F65 and
F130 groups) had higher plasma levels of hOPN (endogenous OPN) at 4 and 6 months of age. Plasma appearance of small amounts of bOPN in the F65 and F130 groups indicates that bOPN was at least partially resistant to digestion and was absorbed. Although the amount of bOPN entering plasma was small and although hOPN derived from breast milk and bOPN in the supplemented formulas differ in their amino acid sequences and post-translational modifications, ${ }^{23,24}$ we hypothesize that the conserved sequence area in the C-terminal containing RGD integrin binding sites ${ }^{25}$ function similarly to increase endogenous OPN production in breast-fed and bOPN-supplemented formula fed infants. The present finding that dietary OPN increased endogenous OPN production is similar to that of our recent study $^{22}$ using KO and WT mice that showed that OPN in milk from wild-type dams is absorbed and stimulates endogenous OPN production in WT pups. Similar results were reported in an animal study, in which endogenous mouse OPN levels in plasma, liver, stomach, and gut were enhanced after adult mice (10 weeks old) were fed bOPN ( $200 \mathrm{mg} / \mathrm{L}$ in $\mathrm{H}_{2} \mathrm{O}$ ) for three weeks. ${ }^{15}$ OPN is a secreted protein ${ }^{2}$ and is primarily expressed in epithelial cells, activated T cells, macrophages, and osteoblasts. ${ }^{26}$ OPN is partly resistant to gastrointestinal digestion and may function systematically. Based on our previous results, after mouse pups (postnatal day 20 ) were fed ${ }^{125}$ I-labeled milk OPN by oral gavage, ${ }^{125}$ I-OPN was detected in various organs including intestine, stomach, heart, and brain. ${ }^{22}$ Endothelial cells and immune cells from blood vessels may be most responsible for contributing to serum OPN concentrations. The effects of dietary OPN on upregulation of endogenous OPN in human and animals need to be further verified in OPN knock-out animals. The previous clinical study findings of a reduction in episodes of fever and levels of the inflammatory cytokine TNF-a in infants receiving breast milk or bOPN-supplemented formula, as opposed to unsupplemented formula, suggest the higher levels of endogenous OPN in those treatment groups have functional systemic benefits. ${ }^{17}$ In the present study, the higher level of plasma hOPN in the BF group relative to the $\mathrm{F} 65$ and $\mathrm{F} 130$ groups at 6 months of age suggest that hOPN is more resistant to digestion and/or more efficiently transferred to the systemic circulation. The variation in infant plasma endogenous OPN concentrations between 1 month and 6 months indicates possible functional effects of different levels of OPN at different developmental stages.

Although breast-feeding is associated with several improved short- and long-term outcomes relative to formula-feeding, ${ }^{27}$ many infants are formula-fed for a variety of reasons. Methods for OPN isolation from milk are well developed, ${ }^{28-30}$ and bovine milk OPN is commercially available and has been found to be safe. ${ }^{16}$ Thus, milk-derived OPN could be an appropriate substitute for human milk OPN in infant formula. 
In summary, we report for the first time OPN concentrations in breast milk throughout 1 year of lactation and effects of orally ingested OPN on plasma OPN levels in infants. Dietary OPN may exert its functions by elevating endogenous OPN concentrations in the circulatory system.

\section{ACKNOWLEDGEMENTS}

This study was supported by an unrestricted grant from Arla Foods Ingredients. The authors acknowledge assistance of Alison Lee for conducting the OPN ELISAs and appreciate Dr. Judith Hills for critical reading of the manuscript.

\section{ADDITIONAL INFORMATION}

Competing interests: The authors declare no competing interests.

Publisher's note: Springer Nature remains neutral with regard to jurisdictional claims in published maps and institutional affiliations.

\section{REFERENCES}

1. Ashkar, S. et al. Eta-1 (osteopontin): an early component of type-1 (cell-mediated) immunity. Science 287, 860-864 (2000).

2. Denhardt, D. T. \& Guo, X. Osteopontin: a protein with diverse functions. FASEB. J. 7, 1475-1482 (1993).

3. Yokosaki, Y. et al. Distinct structural requirements for binding of the integrins alphavbeta6, alphavbeta3, alphavbeta5, alpha5beta1 and alpha9beta1 to osteopontin. Matrix Biol. 24, 418-427 (2005).

4. Barry, S. T. et al. A regulated interaction between alpha5beta1 integrin and osteopontin. Biochem. Biophys. Res. Commun. 267, 764-769 (2000).

5. Green, P. M. et al. Structural elements of the osteopontin SVVYGLR motif important for the interaction with alpha(4) integrins. FEBS Lett. 503, 75-79 (2001)

6. Oldberg, A., Franzen, A. \& Heinegard, D. Cloning and sequence analysis of rat bone sialoprotein (osteopontin) CDNA reveals an Arg-Gly-Asp cell-binding sequence. Proc. Natl Acad. Sci. USA 83, 8819-8823 (1986).

7. Katagiri, Y. U., Sleeman, J. \& Fujii, H. CD44 variants but not CD44s cooperate with beta1-containing integrins to permit cells to bind to osteopontin independently of arginine-glycine-aspartic acid, thereby stimulating cell motility and chemotaxis. Cancer Res. 59, 219-226 (1999).

8. Weber, G. F. et al. Receptor-ligand interaction between CD44 and osteopontin (Eta-1). Science 271, 509-512 (1996).

9. Rangaswami, H., Bulbule, A. \& Kundu, G. C. Osteopontin: role in cell signaling and cancer progression. Trends Cell Biol. 16, 79-87 (2006).

10. Sodek, J., Ganss, B. \& McKee, M. D. Osteopontin. Crit. Rev. Oral. Biol. Med. 11, 279-303 (2000).

11. Schack, L. et al. Considerable variation in the concentration of osteopontin in human milk, bovine milk, and infant formulas. J. Dairy Sci. 92, 5378-5385 (2009).

12. Bruun, S. et al. Osteopontin levels in human milk vary across countries and within lactation period: data from a multicenter study. J. Pediatr. Gastroenterol. Nutr. 67, 250-256 (2018)
13. Lönnerdal, B. Bioactive proteins in human milk: health, nutrition, and implications for infant formulas. J. Pediatr. 173, Suppl:S4-9 (2016).

14. Chatterton, D. et al. In vitro digestion of novel milk protein ingredients for use in infant formulas: Research on biological functions. Trends Food Sci. Technol. 15, 373-383 (2004)

15. Ge, X. et al. Milk osteopontin, a nutritional approach to prevent alcoholinduced liver injury. Am. J. Physiol. Gastrointest. Liver Physiol. 304, G929-939 (2013).

16. Kvistgaard, A. S. et al. Pre-clinical in vitro and in vivo safety evaluation of bovine whey derived osteopontin, Lacprodan(R) OPN-10. Food Chem. Toxicol. 73, 59-70 (2014).

17. Lönnerdal, B. et al. Growth, nutrition, and cytokine response of breast-fed infants and infants fed formula with added bovine osteopontin. J. Pediatr. Gastroenterol. Nutr. 62, 650-657 (2016).

18. West, C. E. et al. Effects of osteopontin-enriched formula on lymphocyte subsets in the first 6 months of life: a randomized controlled trial. Pediatr. Res. 82, 63-71 (2017).

19. Da Silva, A. P. et al. Exacerbated tissue destruction in DSS-induced acute colitis of OPN-null mice is associated with downregulation of TNF-alpha expression and non-programmed cell death. J. Cell. Physiol. 208, 629-639 (2006).

20. Buccigrossi, V. et al. Lactoferrin induces concentration-dependent functional modulation of intestinal proliferation and differentiation. Pediatr. Res. 61 410-414 (2007).

21. Donovan, S. M. et al. Bovine osteopontin modifies the intestinal transcriptome of formula-fed infant rhesus monkeys to be more similar to those that were breastfed. J. Nutr. 144, 1910-1919 (2014).

22. Jiang, R., Prell, C. \& Lönnerdal, B. Milk osteopontin promotes brain development by up-regulating osteopontin in the brain in early life. FASEB. J. 33 (2019) [Epub ahead of print].

23. Sørensen, E. S., Højrup, P. \& Petersen, T. E. Posttranslational modifications of bovine osteopontin: identification of twenty-eight phosphorylation and three Oglycosylation sites. Protein Sci. 4, 2040-2049 (1995).

24. Christensen, B. et al. Post-translationally modified residues of native human osteopontin are located in clusters: identification of 36 phosphorylation and five O-glycosylation sites and their biological implications. Biochem. J. 390, 285-292 (2005).

25. Christensen, B. et al. C-terminal modification of osteopontin inhibits interaction with the alphaVbeta3-integrin. J. Biol. Chem. 287, 3788-3797 (2012).

26. Denhardt, D. T., Giachelli, C. M. \& Rittling, S. R. Role of osteopontin in cellular signaling and toxicant injury. Annu. Rev. Pharmacol. Toxicol. 41, 723-749 (2001).

27. Victora, C. G. et al. Breastfeeding in the 21st century: epidemiology, mechanisms, and lifelong effect. Lancet 387, 475-490 (2016).

28. Azuma, N. et al. A rapid method for purifying osteopontin from bovine milk and interaction between osteopontin and other milk proteins. Int. Dairy J. 16 370-378 (2006).

29. Sørensen, E. S. \& Petersen, T. E. Purification and characterization of three proteins isolated from the proteose peptone fraction of bovine milk. J. Dairy. Res. 60 189-197 (1993).

30. Bayless, K. J., Davis, G. E. \& Meininger, G. A. Isolation and biological properties of osteopontin from bovine milk. Protein Expr. Purif. 9, 309-314 (1997). 